\title{
Eye Fundus
}

National Cancer Institute

\section{Source}

National Cancer Institute. Eye Fundus. NCI Thesaurus. Code C135172.

The interior surface of the eye opposite the lens including the retina, optic disc, macula, fovea, and posterior pole. 